\title{
Infantile Dural Arteriovenous Fistula of the Transverse Sinus Presenting with Ocular Symptoms, Case Reports and Review of Literature
}

\author{
Ahmed Elsayed Sultan, M.D., Ph.D., Tamer Hassan, M.D., Ph.D. \\ Department of Neurosurgery, Alexandria University School of Medicine, Alexandria, Egypt
}

Dural arteriovenous fistula (DAVF) of the transverse sinus with ophthalmic manifestations in young children are rare. We reviewed two cases of direct AVF of the transverse sinus with ocular manifestations managed at our institution. The first, a 2.5 years old male child presented with left exophthalmos. Angiography revealed AVF between the occipital artery and the transverse sinus. The second, a 2 years old female child, complained of left exophthalmos. Imaging studies showed bilateral direct AVFs of the transverse sinus with bilateral dysmaturation of the sigmoid sinus. Transarterial embolization was done in both cases. Clinical and radiological follow up revealed complete cure. This report suggests that DAVF of the transverse sinus supplied by the external carotid branches can present with ophthalmic manifestations especially if there is distal venous stenosis or obliteration involving sigmoid sinus. Transarterial embolization using coils and liquid embolic agents could be safe and feasible to obliterate the fistula.

Key Words : Dural arteriovenous fistula $\cdot$ Exophthalmos · Transverse sinus $\cdot$ Sigmoid sinus dysmaturation.

\section{INTRODUCTION}

Dural arteriovenous fistulas (DAVFs) are abnormal vascular connections consisting abnormally of one or more meningeal feeding arteries that drain directly into a venous sinus or intracranial vein $^{1,4,7,10,18)}$.

DAVFs can be found in any age group but they are mostly acquired lesions that present in the middle or late in life. Distal sinus occlusion or stenosis plays a major role behind fistula formation. Dural sinus thrombosis and inflammation play an important role in the development of acquired DAVFs. Pediatric DAVFs may be congenital malformation of the sinus that present early in neonatal or infantile period, or acquired lesions like those presenting late in life ${ }^{13,14,16,17)}$.

The pattern of venous drainage and the location of the fistula are the main factors affecting clinical presentation, also the presence of associated venous hypertension ${ }^{17)}$. The range of presentations reported include audible bruit, headache, seizures, progressive neurological deficit, cerebellar symptoms and heart failure $^{2,6,15,16)}$. The risk of intra-cerebral hemorrhage increases markedly with the retrograde venous drainage, venous hyper- tension, and cortical venous reflux ${ }^{2,5,16)}$. Drainage through the ophthalmic vein may cause ocular symptoms which include engorged vessels of the sclera, chemosis, proptosis, increase intraocular pressure and secondary glaucoma, diplopia, periorbital edema, limitation of extraocular muscle movement, and papillaedema ${ }^{2,5,6,15,16,19)}$. The severity of the ophthalmic symptoms might be related to the location and duration of intracranial DAVF which in turn affects the degree of venous drainage through the ophthalmic veins ${ }^{2,9,19)}$. In general, posteriorly located DAVFs have fewer ocular symptoms than anterior ones ${ }^{16,17,19)}$.

We recently encountered two young children with exophthalmos resulting from direct fistula between the dural branches of the external carotid artery (ECA) and the transverse sinus, and both cases have retrograde venous drainage back to the cavernous sinus then to the ophthalmic veins.

\section{CASE REPORT}

\section{Patient 1}

A Male child aged 2.5 year, had an orbital swelling of the left eye since birth with slow progressive course. On physical exami-

- Received : December 5, 2014 • Revised : December 22, 2014 • Accepted : March 9, 2015

- Address for reprints : Ahmed Elsayed Sultan, M.D., Ph.D.

Lecturer of Neurosurgery, Endovascular Neurosurgeon, Neurosurgery Department Faculty of Medicine, Alexandria University, Alexandria Faculty of medicine, Champollion

Street, El-Khartoum Square, Azarita Medical Campus, Alexandria 21131, Egypt

Tel : +20-1001042419, Fax : +20-34862506, E-mail : ahmed.sultan@alexmed.edu.eg

- This is an Open Access article distributed under the terms of the Creative Commons Attribution Non-Commercial License (http://creativecommons.org/licenses/by-nc/3.0) which permits unrestricted non-commercial use, distribution, and reproduction in any medium, provided the original work is properly cited. 
nation there was only exophthalmos with conjunctival congestion. MRI brain confirmed the presence of dilated tortuous cavernous sinus and left superior ophthalmic vein. CT angiography was done and it showed direct fistulous communication between left ECA branches and left transverse sinus with venous reflux into the deep venous system, cavernous sinus, and left superior ophthalmic vein. DSA revealed direct fistula of the transverse sinus supplied mainly by a hugely dilated occipital artery with minimal supply from the dural branches of left vertebral artery. Dysmaturation of the sigmoid sinus distal to the fistulous communication explained the occurrence of retrograde deep venous reflux, superior ophthalmic vein varicosity and exophthalmos. The DAVF of this patient was classified as type IIa according to Cognard's classification because of the retrograde flow through the sinus but with no retrograde flow into cortical veins. Endovascular embolization was performed with the patient under general anesthesia. A transarterial transfemoral approach was chosen. A 5 Fr Envoy guiding catheter (Cordis Neurovascular, Miami Lakes, FL, USA) was placed in the ECA after confirmation of the fistula site by DSA. An Excelsior ${ }^{\circledR}$ SL- $10^{\circledR}$ microcatheter (Boston Scientific, Natick, MA, USA) was subsequently navigated over Transend 10 wire (Boston Scientific, Natick, MA, USA) to reach the site of the fistula at the left transverse sinus. Superselective angiography was then performed to confirm optimal position. The microcatheter was flushed continuously with hepranized normal saline. Guglielmi Detachable Coils (GDC; Boston Scientific Corporation, Natick, MA, USA) were introduced with simultaneous mechanical external compression of the occipital artery to decrease the flow in the fistula to allow for settling of the coils. Coiling is continued until disappearance of the flow inside the fistula. Then the microcatheter was flushed with 25\% dextrose solution. Then N-butyl Cyanoacrylate (NBCA) at 50\% concentration was used to achieve complete obliteration of the fistula. Control angiography was done at the end of the procedure to confirm obliteration of the fistula. The patient recovered completely without deficits. The ocular manifestation disappeared at 3 months follow up. Two years after embolization clinical and radiological follow up using DSA showed permanent obliteration of the fistula (Fig. 1,2).

\section{Patient 2}

A female child aged 1.5 years, had an exophthalmos, con-
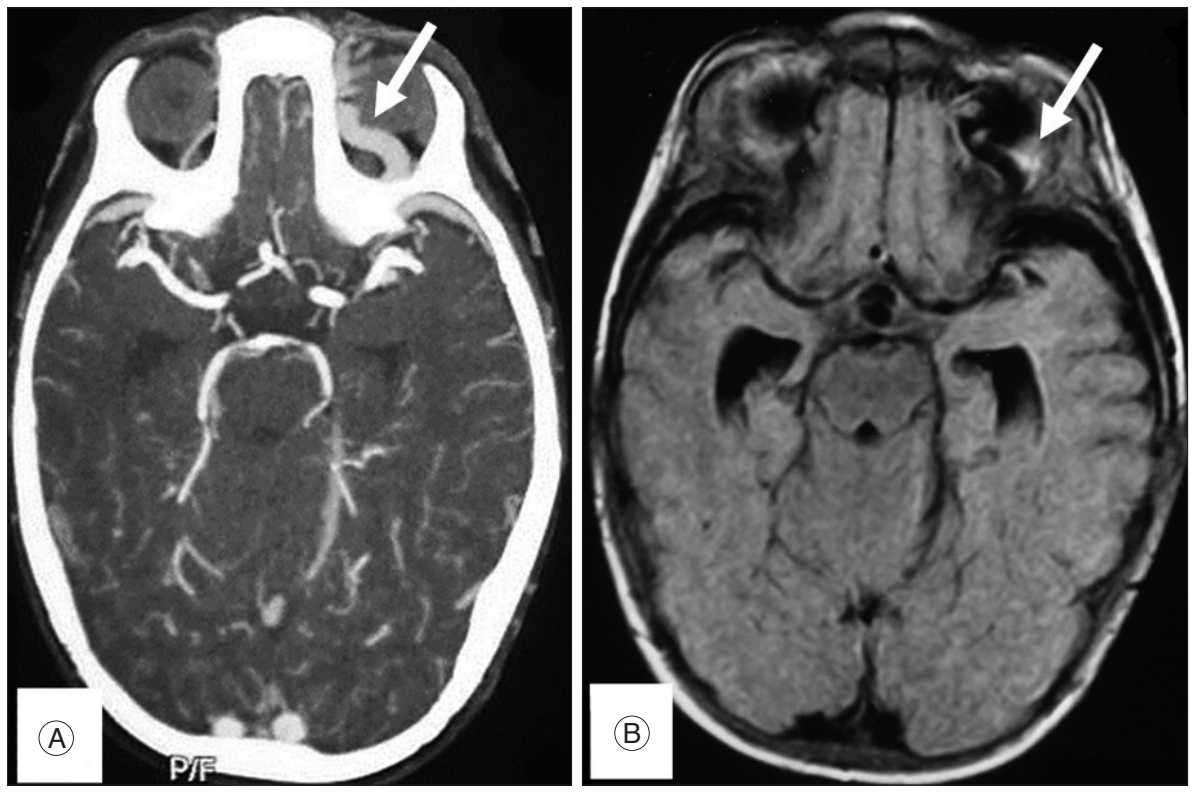

Fig. 1. Imaging study of case 1 before embolization showing direct fistula with the transverse sinus. A : Axial view of brain CTA showing enlarged left superior ophthalmic vein (arrow). B : Axial T1 MRI brain showing enlarged left superior ophthalmic vein (arrow). C and D : 3D CTA showing the fistula between the occipital artery and the transverse sinus enlarged left superior ophthalmic vein (arrow).
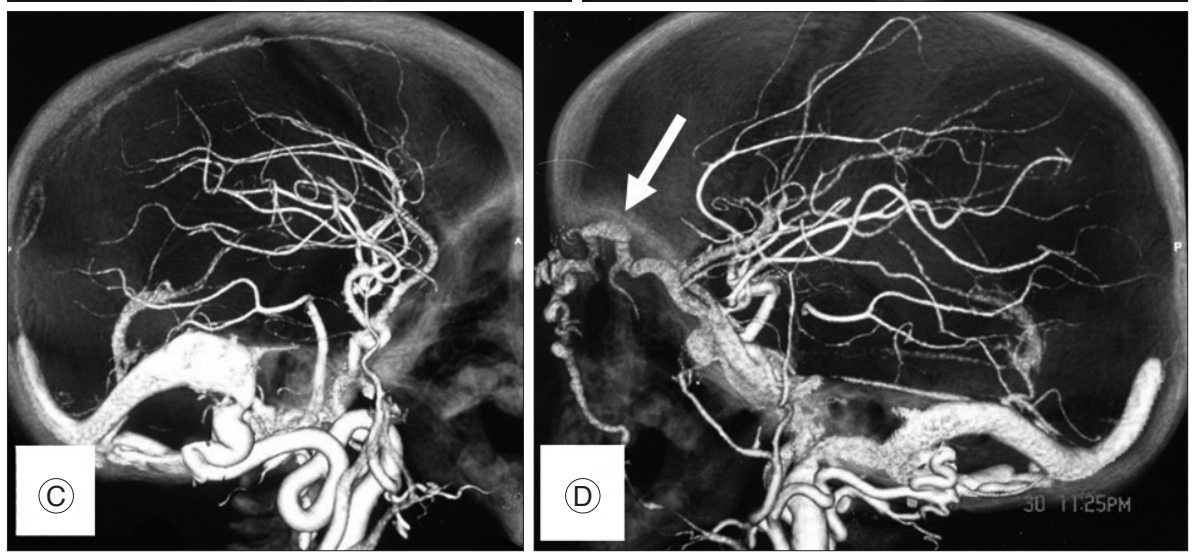

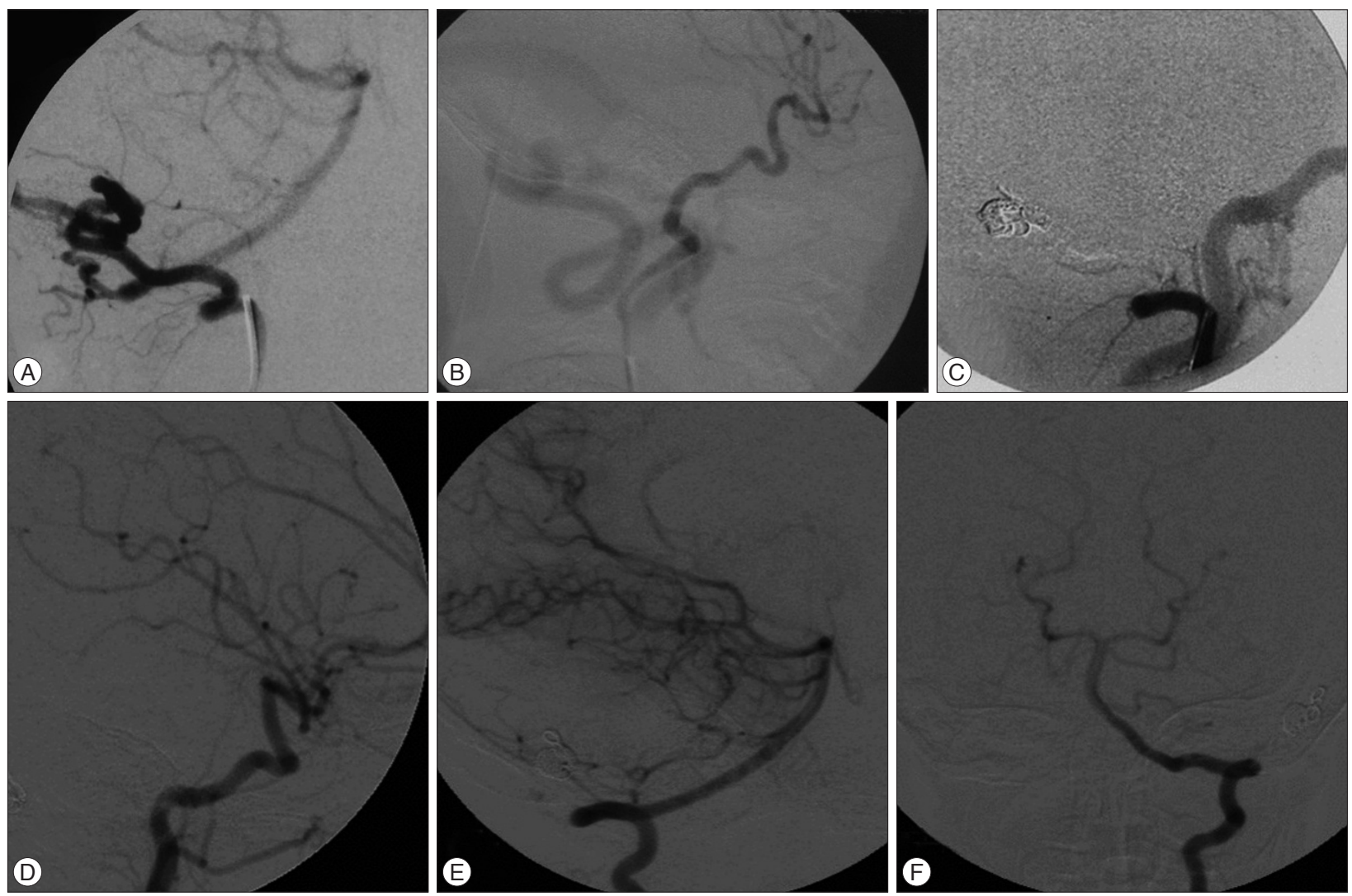

Fig. 2. Imaging study of case 1 before and after embolization showing direct fistula with the transverse sinus. A : Lateral view of the left vertebral artery showing vertebral dural branches supplying the fistula. B : Lateral view of left common carotid injection showing a hugely dilated occipital artery directly communicating with the transverse sinus. C : Common carotid injection after coiling and NBCA injection showing occlusion of the fistula. D : Lateral view of the left CCA angiography after 2 years showing disappearance of the fistula. E and $F$ : anteroposterior and lateral view of the left vertebral artery angiogram showing disappearance of the fistula. NBCA : N-butyl Cyanoacrylate, CCA : common carotid artery.
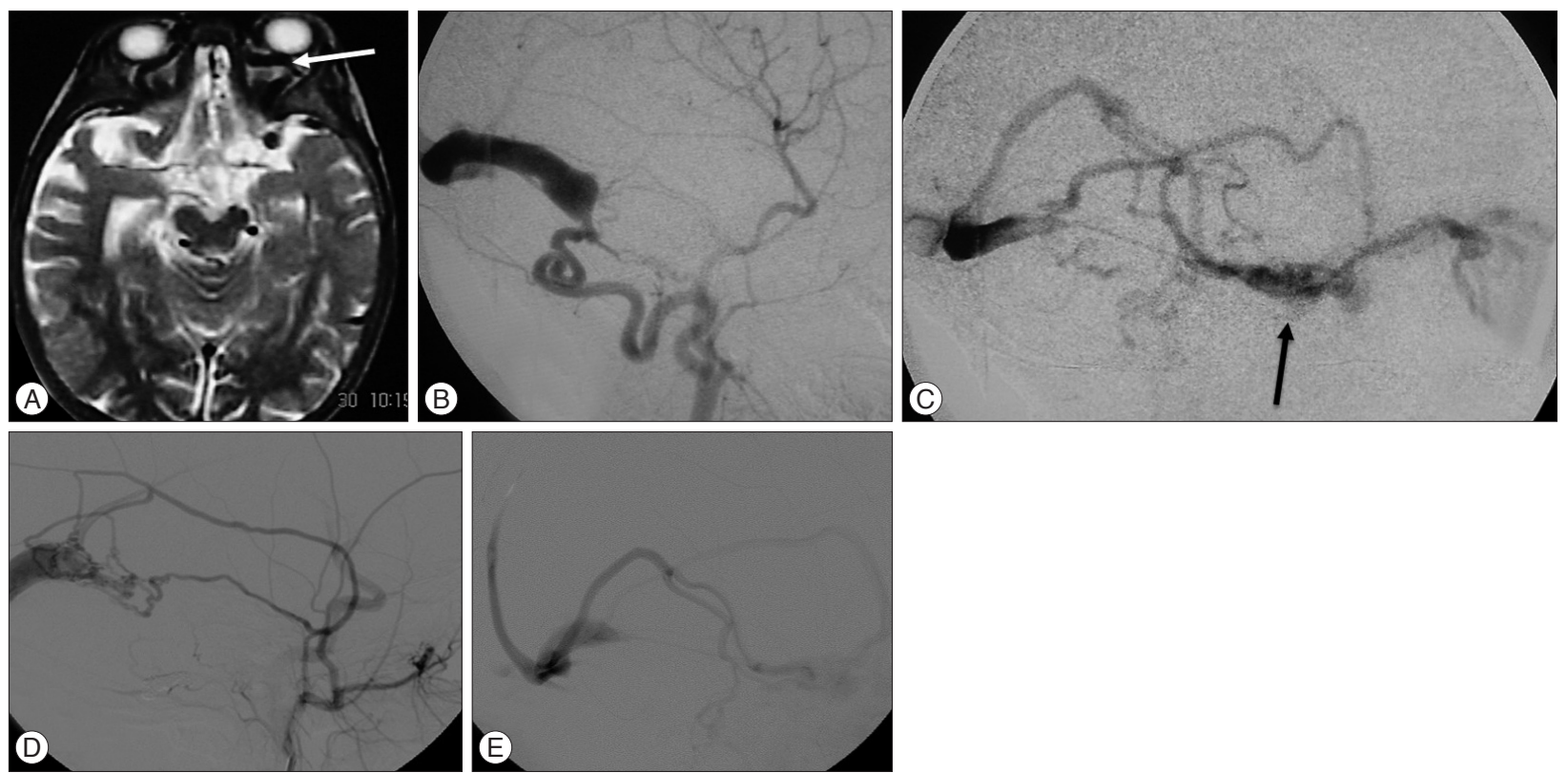

Fig. 3. Imaging study of case 2, a 1.5 years old female patient presenting with proptosis. A : Axial T2 MRI of the brain showing dilated ophthalmic veins (white arrow). B : Right CCA angiogram showing direct fistula between the occipital artery and the transverse sinus with the absence of the sigmoid sinus. C : Venous phase of right CCA angiogram showing venous reflux anteriorly through the deep and superficial venous systems including the cavernous sinus (black arrow) and ophthalmic veins. D : Lateral view of left CCA angiogram showing AVF supplied by branches of the ECA. E : Venous phase of left CCA angiogram showing retrograde venous drainage into the superficial and deep venous systems with absent sigmoid sinus. CCA : common carotid artery, ECA : external carotid artery, AVF : arteriovenous fistula. 
junctival congestion, and eye lid varices since birth. MRI brain showed dilated and tortuous left ophthalmic veins. Endovascular intervention was decided under general anesthesia. Transfemoral DSA revealed bilateral direct fistulous communication between the left transverse sinus and the ECA branches. We observed bilateral dysmaturation of the sigmoid sinus that explained the retrograde venous reflux into the superficial and deep venous systems. The DAVF of this patient was classified as type IIb according to Cognard's classification because of the retrograde venous reflux through the sinus and cortical veins. The

Fig. 4. Cerebral angiography during embolization of the fistula of case 2. A : Lateral view of right ECA angiogram showing AVF supplied by occipital artery. B : Occlusion of the fistula using coils and NBCA. C : Lateral view of left ECA angiogram showing AVF supplied by branches of the ECA. D : Left ECA angiogram after Onyx embolization showing complete disappearance of the fistula. ECA : external carotid artery, AVF : arteriovenous fistula, NBCA : N-butyl Cyanoacrylate.

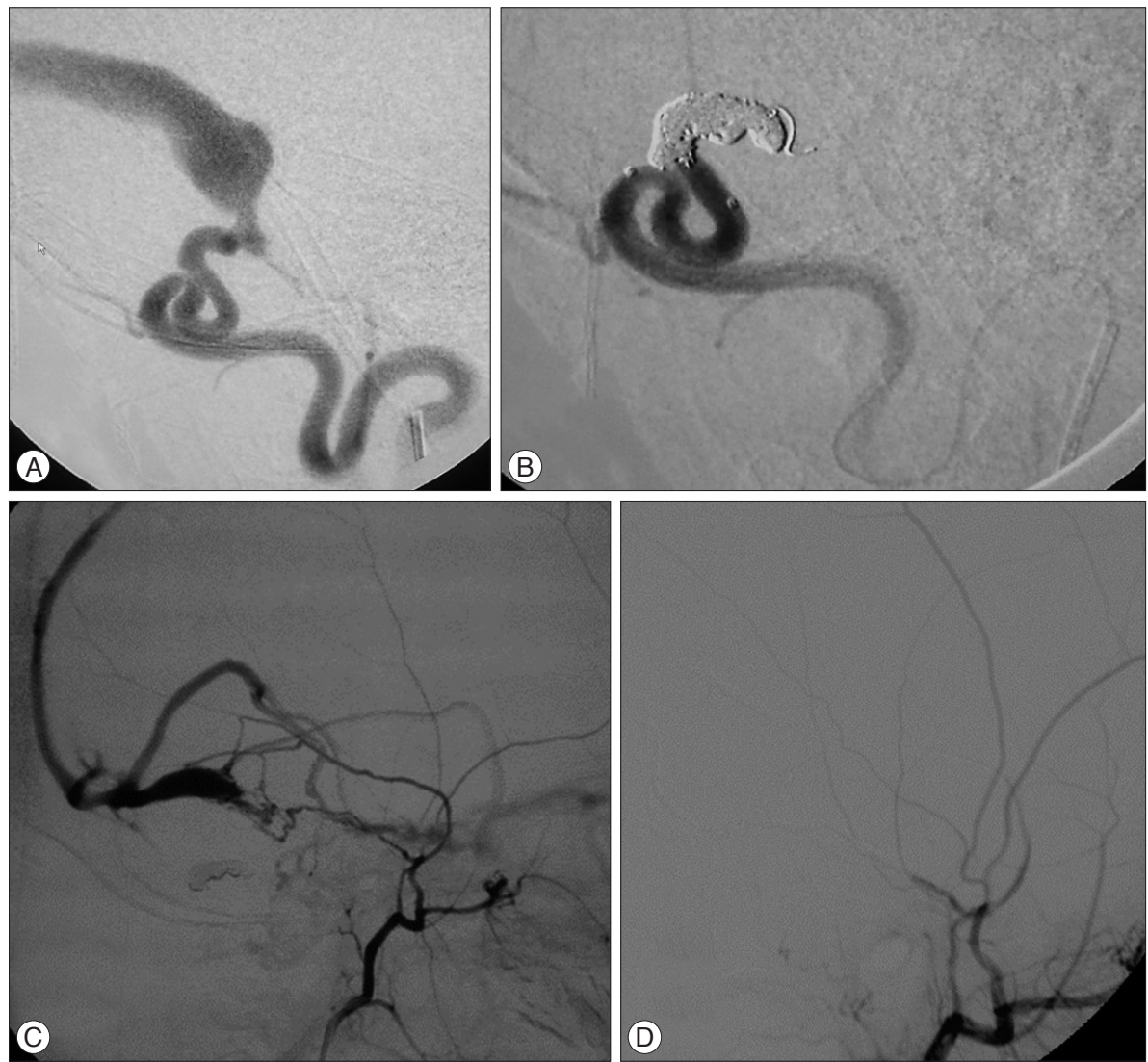

Fig. 5. Cerebral angiography 4 years after embolization in case 2. A : Lateral view of left CCA angiogram showing complete disappearance of the fistula. $B$ : Lateral views of the venous phase of left CCA angiogram showing retrograde venous drainage anteriorly into the superficial and deep venous systems with absent sigmoid sinus with complete fistula disappearance (arrow). $C$ : Venous phase of right CCA angiogram showing drainage is passing through the cavernous sinus and ophthalmic with absent sigmoid sinus with complete fistula disappearance (arrow) $\mathrm{D}$ : Plain X-ray lateral view of the skull showing shadow of Onyx and coils. CCA common carotid artery.

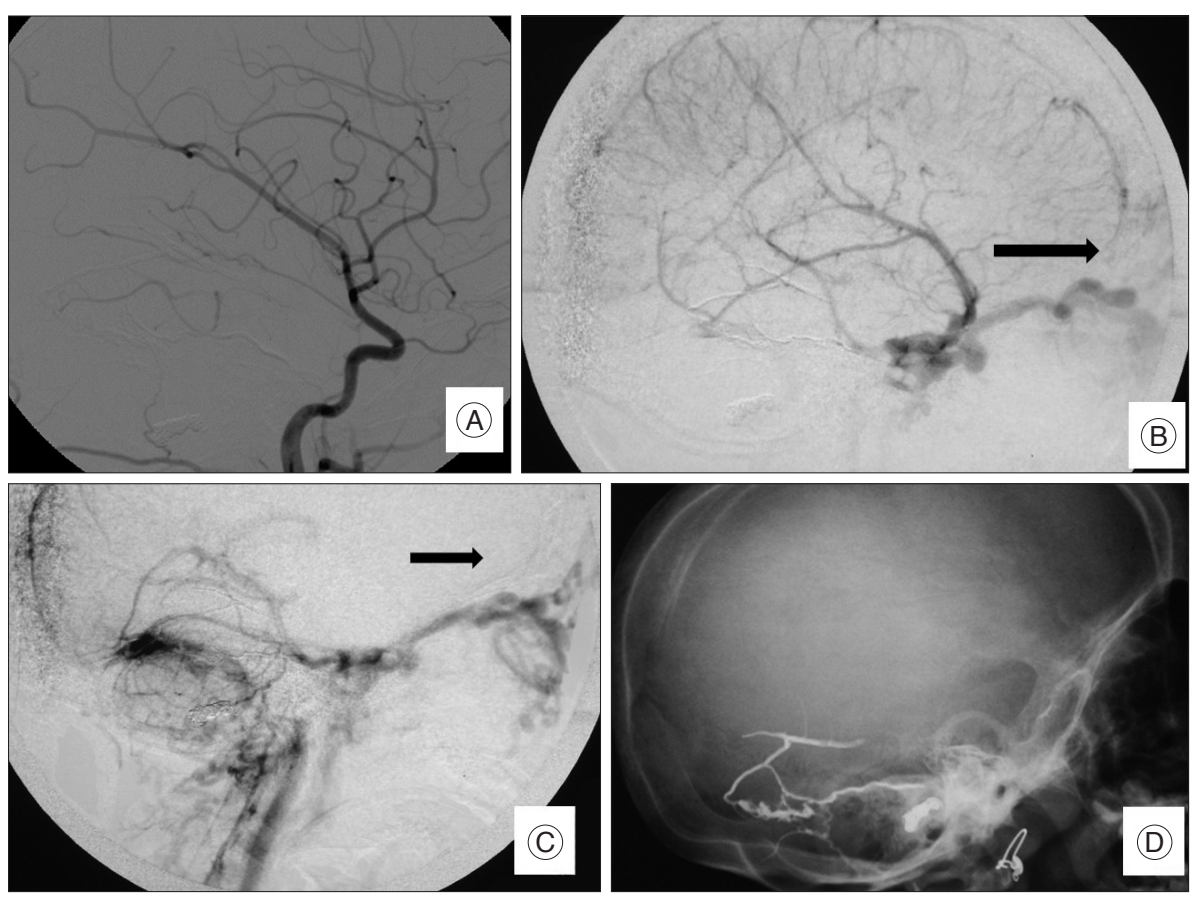


two fistulas were embolized over two sessions due to dye volume limitation in this young child. In the first session catheterization of the right ECA was performed via a transfemoral approach using standard coaxial techniques and with the catheter subject to continuous flushing with heparinized saline (4000 U of heparin per liter). A 4 Fr femoral sheath was introduced, followed by introduction of a 4 Fr catheter into the right ECA. An Excelsior $^{\circledR}$ SL-10 ${ }^{\circledR}$ microcatheter (Boston Scientific) was subsequently navigated over Transend 10 wire (Boston Scientific) through the right occipital artery to reach the fistula site. Superselective angiography was then performed to confirm optimal position. GDC (Boston Scientific Corporation) were introduced with simultaneous mechanical external compression of the occipital artery to decrease the flow in the fistula in order to allow the coils to settle in their desired location. Coiling was continued until disappearance of the flow inside the fistula. Then the microcatheter was flushed with $25 \%$ dextrose solution, and 50\% NBCA was injected to fully obliterate the fistula. DSA was done at the end of the procedure to confirm obliteration of the fistula. The ocular manifestation disappeared partially after 3 month. The exophthalmos decreased but some orbital varicosities remained. A 2nd sessions was done after 6 months to occlude the left sided fistula. Under general anesthesia, a 5 Fr sheath was introduced through the right femoral artery. A 5 Fr Envoy guiding catheter (Cordis Neurovascular, Miami Lakes, FL, USA) was introduced through the left ECA. A 1.5 Fr Marathon microcatheter (Covidien, Irvine, CA, USA) microcatheter was navigated through the middle meningeal artery to reach the fistula then superselective angiogram was done. After reaching the optimum site and preparing the microcatheter using dimethyl-sulfoxide, Onyx-18 (Covidien, Irvine, CA, USA) was infused until complete obliteration. Final DSA revealed complete fistulous obliteration bilaterally, but the retrograde venous drainage through the whole venous system was observed due bilateral occlusion of the sigmoid sinuses. DSA follow up after 4 years revealed complete obliteration of the fistula, absence of the sigmoid sinus bilaterally, and the final venous drainage rerouted through the prevertebral, paravertebral, pterygoid venous plexus, and ophthalmic veins. Clinical follow up of this patient revealed presence of eye lid varicosities but no proptosis (Fig. 3, 4, 5).

\section{DISCUSSION}

Most of DAVFs are acquired and occur mostly after the 4th decade. Some lesions are reported to be congenital which are usually large direct shunts ${ }^{4,13,18,19)}$. The pathogenesis of DAVF is controversial. Various factors may contribute to their formation, such as sinus thrombosis, trauma or tumor invasion ${ }^{8,18,19)}$. The clinical presentations are related to the location of the fistula and the pattern of venous drainage ${ }^{12,17)}$.

Ocular manifestation are related to the location and duration of the fistula, these manifestations occur mainly with anteriorly located fistulas ${ }^{17,19)}$. In general, the more posterior the fistula, the fewer the ocular symptoms noted, and reported cases of associated ocular manifestations with posterior fossa DAVF are rare ${ }^{19)}$. To our knowledge there are no reported cases of congenital direct AVF between the ECA and transverse sinus that present with proptosis. All reported cases are acquired and many arterial feeders are encountered from ECA and VA, the venous sinus involved included transverse, occipital, marginal, and IPS.

Liu et al. ${ }^{11)}$ reported 2 cases of posterior fossa DAVFs presenting with exophthalmos, chemosis, and eyelid swelling resulting from retrograde venous drainage into the cavernous sinus and superior ophthalmic vein but the transverse sinus was not involved. The retrograde venous reflux passes through either the SPS or the cortical veins of the temporal lobe to the Sylvian veins to the cavernous sinus then to the ophthalmic veins.

McDougall et al. ${ }^{12)}$ reported 14 adult patients with marginal sinus DAVF; One of these patients also had diplopia, chemosis, and proptosis due to retrograde venous drainage up the inferior petrosal sinus. Similarly, a second patient had chemosis but without proptosis or diplopia i.e., only 2 patients has ocular abnormalities related to posterior fossa DAVF again the transverse sinus was not involved.

Outlet obstruction increases the retrograde venous reflux; one of our cases (patient one) had dysmaturation of the sigmoid sinus which increased the venous reflux and the 2 nd case had bilateral congenital obliteration of the sigmoid sinus. Mironov ${ }^{13)}$ reported a case of inferior petrosal sinus DAVF presenting with contralateral exophthalmos which had distal obstruction of the IPS resulting in retrograde venous reflux into the cavernous sinus and ophthalmic veins. In our 2 nd case the larger fistula was on the right side and the proptosis affected the left eye.

Endovascular treatment using platinum coils followed by glue used in our cases was an effective method in occlusion of DAVF. Liu et al. ${ }^{11)}$ used the platinum coils and NBCA effectively in embolization of DAVFs. In our cases we used coils plus liquid embolic agents to obliterate the fistulae.

DAVFs usually develops after sinus occlusion due congenital atresia or acquired causes. Complete sinus occlusion results in interruption of the venous outflow and redirection of the venous drainage into the deep or superficial systems ${ }^{3)}$. Cortical venous reflux is dangerous and results in intracranial hemorrhage. Retrograde venous drainage into the cavernous sinus, ophthalmic veins, emissary veins and vertebral venous plexuses represents an alternate pathway for sigmoid sinus dysmaturation. This was found typically in case two. It is important not to interrupt the anterior venous pathway during embolization in cases with occlusion or dysmaturation of the sigmoid sinus, as this anterior drainage takes up the normal venous drainage of the brain.

\section{CONCLUSION}

This report suggests that DAVF of the transverse sinus sup- 
plied by the external carotid branches can present with ophthalmic manifestations especially if there is distal venous stenosis or obliteration involving sigmoid sinus. Transarterial embolization using coils and liquid embolic agents could be safe and feasible to obliterate the fistula.

\section{References}

1. Berenstein A, Lasjaunias PL, TerBrugge KG : Dural arteriovenous shunts. Surgical Neuroangiography. Heidelberg : Springer-Verlag Berlin Heidelberg, Vol 3 : Clinical and Interventional Aspects in Children, 2006, pp389-453

2. Borden JA, Wu JK, Shucart WA : A proposed classification for spinal and cranial dural arteriovenous fistulous malformations and implications for treatment. J Neurosurg 82 : 166-179, 1995

3. Cataltepe O, Berker M, Gürçay O, Erbengi A : An unusual dural arteriovenous fistula in an infant. Neuroradiology 35 : 394-397, 1993

4. Chaudhary MY, Sachdev VP, Cho SH, Weitzner I Jr, Puljic S, Huang YP : Dural arteriovenous malformation of the major venous sinuses : an acquired lesion. AJNR Am J Neuroradiol 3 : 13-19, 1982

5. Cognard C, Gobin YP, Pierot L, Bailly AL, Houdart E, Casasco A, et al. : Cerebral dural arteriovenous fistulas : clinical and angiographic correlation with a revised classification of venous drainage. Radiology 194 : 671-680, 1995

6. Davies MA, TerBrugge K, Willinsky R, Coyne T, Saleh J, Wallace MC : The validity of classification for the clinical presentation of intracranial dural arteriovenous fistulas. J Neurosurg 85 : 830-837, 1996

7. Harrigan MR, Deveikis JP, Ardelt AA : Dural arteriovenous fistulas in Harrigan MR, Deveikis JP (eds) : Handbook of Cerebrovascular Disease and Neurointerventional Technique. New York : Humana Press, 2009, pp539-560

8. Herman JM, Spetzler RF, Bederson JB, Kurbat JM, Zabramski JM : Genesis of a dural arteriovenous malformation in a rat model. J Neurosurg 83 : 539-545, 1995

9. Houdart E, Gobin YP, Casasco A, Aymard A, Herbreteau D, Merland JJ :
A proposed angiographic classification of intracranial arteriovenous fistulae and malformations. Neuroradiology $35: 381-385,1993$

10. Ihn YK, Kim MJ, Shin YS, Kim BS : Dural arteriovenous fistula involving an isolated sinus treated using transarterial onyx embolization. J Korean Neurosurg Soc 52 : 480-483, 2012

11. Liu HM, Shih HC, Huang YC, Wang YH : Posterior cranial fossa arteriovenous fistula with presenting as caroticocavernous fistula. Neuroradiology 43 : 405-408, 2001

12. McDougall CG, Halbach VV, Dowd CF, Higashida RT, Larsen DW, Hieshima GB : Dural arteriovenous fistulas of the marginal sinus. AJNR Am J Neuroradiol 18 : 1565-1572, 1997

13. Mironov A : Dural arteriovenous fistula of the inferior petrosal sinus producing contralateral exophthalmus. Neuroradiology 36 : 619-621, 1994

14. Morita A, Meyer FB, Nichols DA, Patterson MC : Childhood dural arteriovenous fistulae of the posterior dural sinuses : three case reports and literature review. Neurosurgery 37 : 1193-1199; discussion 1199-1200, 1995

15. Nakamura M, Tamaki N, Hara Y, Nagashima T : Two unusual cases of multiple dural arteriovenous fistulas. Neurosurgery 41 : 288-292; discussion 292-293, 1997

16. Souza MP, Willinsky RA, Terbrugge KG : Intracranial dural arteriovenous shunts in children. The toronto experience. Interv Neuroradiol 9 (Suppl 2) : 47-52, 2003

17. Spittau B, Millán DS, El-Sherifi S, Hader C, Singh TP, Motschall E, et al. : Dural arteriovenous fistulas of the hypoglossal canal : systematic review on imaging anatomy, clinical findings, and endovascular management. J Neurosurg 122 : 883-903, 2015

18. Toledo MM, Wilson TJ, Dashti S, McDougall CG, Spetzler RF : Dural arteriovenous fistula associated with superior sagittal sinus occlusion secondary to invasion by a parafalcine meningioma : case report. Neurosurgery 67 : 205-207; discussion 207, 2010

19. Wang TJ, Jou JR, Woung LC, Shih YF, Lin LLK : Ophthalmic manifestations of intracranial dural arteriovenous fistula - report of four cases. Tzu Chi Med J 17 : 93-99, 2005 